\title{
Galactic disk structure as revealed by LAMOST A stars
}

\author{
B.-Q. Chen, ${ }^{1,4}$ X.-W. Liu, ${ }^{1}$ H.-B. Yuan, ${ }^{2}$ Y. Huang, ${ }^{1,4}$ M.-S. Xiang, ${ }^{3,4}$ \\ C. Wang, ${ }^{1}$ Z.-J. Tian ${ }^{1,4}$ and H.-W. Zhang ${ }^{1}$ \\ ${ }^{1}$ Department of Astronomy, Peking University, Beijing 100871, P. R. China \\ email: bchen@pku.edu.cn \\ ${ }^{2}$ Department of Astronomy, Beijing Normal University, Beijing 100875, P. R. China \\ ${ }^{3}$ National Astronomy Observatories, Chinese Academy of Sciences, Beijing 100012, P. R. China \\ ${ }^{4}$ LAMOST fellow
}

\begin{abstract}
Using the spectroscopic distances of over 0.12 million A-type stars selected from the LAMOST Spectroscopic Survey of the Galactic Anti-center (LSS-GAC), we map their threedimensional number density distributions in the Galaxy. These stellar number density maps allow an investigation of the Galactic young age thin disk structure with no a priori assumptions about the functional form of its components. The data show strong evidence for a significant flaring young disk. A more detail analysis show that the stellar flaring have different behaviours between the Northern and the Southern Galactic disks. The maps also reveal spatially coherent, kpc-scale stellar substructure in the thin disk. Finally, we detect the Perseus arm stellar overdensity at R $\sim 10 \mathrm{kpc}$.
\end{abstract}

Keywords. Galaxy: disk, Galaxy: structure

\section{Overview}

The Milky Way is the only galaxy that we can study its structure using the stellar number densities. However the study is hampered by the heavy interstellar dust extinction and the difficulty of obtaining accuratedistances to individual stars. To study the Galactic thin disk, which has the strongest extinction and owns stars of a large range of ages, is particularly difficult.

Samples of stars with limited age range and accurate distance estimates can be used to avoid the problems discussed above. In this work we select a sample of A stars from the LSS-GAC ( $\mathrm{Liu}$ et al. 2014) value-added catalogue, which contains data observed by LAMOST since June 2016 (Xiang et al. 2017b). In total, over 0.12 million A stars are selected. Absolute magnitude and stellar atmospheric parameters of these stars are estimated with the LAMOST Stellar Parameter Pipeline at Peking University (LSP3, Xiang et al. 2017a). Values of the interstellar extinction are obtained for individual stars with the standard pairing technique (Yuan et al. 2015). The selection effects of LSSGAC are corrected similarly to the work of (Chen et al. 2017). To test the spectroscopic distances of stars in our sample, we compare them with with the Gaia-TGAS parallaxes (Gaia Collaboration et al. 2016). Our spectroscopic distances are in good agreement with the Gaia TGAS parallax, with a TGAS-based distance error smaller than $20 \%$.

\section{The Galactic thin disk (sub)structure}

We have obtained the averaged stellar number density maps of our sample. The stellar density distributions for different ranges of $\phi$ show similar features. They do not show simplicity in agreement with a single exponential disk model. Significant stellar 

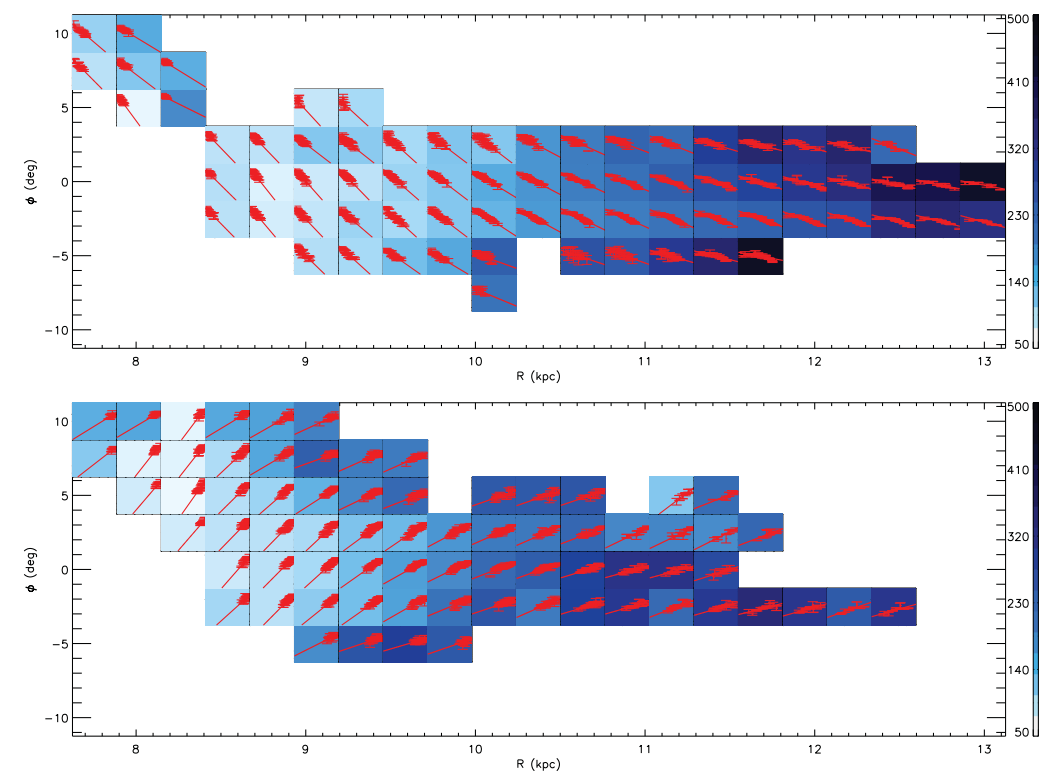

Figure 1. Disk flaring (color scale) derived with our sample for stars in the Northern (upper panel) and Southern (bottom panel) Galactic disks, respectively. Each sub-panel plots stellar density as a function of $Z$ (points with error bars) along with a linear fit to the data (straight lines). The vertical axis of each sub-panel ranges $\phi$ from -10.5 to $10.5 \mathrm{deg}$ and the horizontal axis $R$ from 7 to $13 \mathrm{kpc}$.

flaring is clearly visible in the outer Galactic disk. There are also moderate North-South asymmetry in the outer part of the disk, starting at $R \sim 9 \mathrm{kpc}$.

Detail analysis show that the stars flare differently between the Northern disk and the Southern disk (Fig. 1). Comparing to the Southern disk, the Northern disk has smaller solar neighbourhood scale height but larger flaring scale. We also find that for $R<$ $9.5 \mathrm{kpc}$, the Southern disk have more stars than the Northern disk, while for $R>9.5 \mathrm{kpc}$ the opposite. This may be a result of the bending-mode perturbations recently identified across the disk plane (Sun et al. 2015). Finally, we detect an overdensity which may be refer to the Perseus Arm at $R \sim 10 \mathrm{kpc}$.

\section{Acknowledgements}

This work is supported by National Key Basic Research Program of China 2014CB845700 and China Postdoctoral Science Foundation 2016M590014.

\section{References}

Chen, B.-Q., et al. 2017, MNRAS, 464, 2545

Gaia Collaboration, Prusti, T., de Bruijne, J. H. J., et al. 2016, A\&AA, 595, A1

Liu, X.-W., et al. 2014, in IAU Symposium, Vol. 298, IAU Symposium, ed. S. Feltzing, G. Zhao,

N. A. Walton, \& P. Whitelock, 310-321

Sun, N.-C., et al. 2015, Research in Astronomy and Astrophysics, 15, 1342

Xiang, M.-S., et al. 2017a, MNRAS, 464, 3657

Xiang, M.-S., et al. 2017b, MNRAS, 467, 1890

Yuan, H.-B., et al. 2015, MNRAS, 448, 855 